\title{
The role of membrane lipids in the induction of macrophage apoptosis by microparticles.
}

Huber, L C ; Jüngel, A ; Distler, J H W ; Moritz, F ; Gay, R E ; Michel, B A ; Pisetsky, D S ; Gay, S ; Distler, O

\begin{abstract}
Microparticles are membrane-derived vesicles that are released from cells during activation or cell death. These particles can serve as mediators of intercellular cross-talk and induce a variety of cellular responses. Previous studies have shown that macrophages undergo apoptosis after phagocytosing microparticles. Here, we have addressed the hypothesis that microparticles trigger this process via lipid pathways. In these experiments, microparticles induced apoptosis in primary macrophage cells or cell lines (RAW 264.7 or U937) with up to a 5-fold increase. Preincubation of macrophages with phosphatidylinositol-3,5-bisphosphate $(\operatorname{PtdIns}(3,5) \mathrm{BP})$ reduced the microparticle-induced apoptosis in a dose-dependent manner. PtdIns $(3,5) \mathrm{BP}$ is a specific inhibitor of the acid sphingomyelinase and thus can block the generation of pro-apoptotic ceramides. Similarly, the pre-incubation of macrophages with Pt$\mathrm{dIns}(3,5) \mathrm{BP}$ prevented microparticle-induced upregulation of caspase 8 , which is a major target molecule of ceramide action in the apoptosis pathway. PtdIns(3,5)BP, however, had no effect on the spontaneous rate of apoptosis. To evaluate further signaling pathways induced by microparticles, the extracellular signal regulated kinase (ERK-) 1 was investigated. This kinase plays a role in activating phospholipases A2 which cleaves membrane phospholipids into arachidonic acid; microparticles have been suggested to be a preferred substrate for phospholipases A2. As shown in our experiments, microparticles strongly increased the amount of phosphorylated ERK1/2 in RAW 264.7 macrophages in a time-dependent manner, peaking 15 min after co-incubation. Addition of PD98059, a specific inhibitor of ERK1, prevented the increase in apoptosis of RAW 264.7 macrophages. Together, these data suggest that microparticles perturb lipid homeostasis of macrophages and thereby induce apoptosis. These results emphasize the importance of biolipids in the cellular cross-talk of immune cells. Based on the fact that in clinical situations with excessive cell death such as malignancies, autoimmune diseases and following chemotherapies high levels of circulating microparticles might modulate phagocytosing cells, a suppression of the immune response might occur due to loss of macrophages.
\end{abstract}

DOI: https://doi.org/10.1007/s10495-006-0622-7

Posted at the Zurich Open Repository and Archive, University of Zurich

ZORA URL: https://doi.org/10.5167/uzh-15998

Journal Article

Published Version

Originally published at:

Huber, L C; Jüngel, A; Distler, J H W; Moritz, F; Gay, R E; Michel, B A; Pisetsky, D S; Gay, S; Distler, O (2007). The role of membrane lipids in the induction of macrophage apoptosis by microparticles. Apoptosis, 12(2):363-374. 
DOI: https://doi.org/10.1007/s10495-006-0622-7 


\title{
The role of membrane lipids in the induction of macrophage apoptosis by microparticles
}

\author{
Lars C. Huber • Astrid Jüngel • Jörg H. W. Distler • Falk Moritz • Renate E. Gay • \\ Beat A. Michel • David S. Pisetsky · Steffen Gay • Oliver Distler
}

Published online: 26 December 2006

(C) Springer Science + Business Media, LLC 2006

\begin{abstract}
Microparticles are membrane-derived vesicles that are released from cells during activation or cell death. These particles can serve as mediators of intercellular crosstalk and induce a variety of cellular responses. Previous studies have shown that macrophages undergo apoptosis after phagocytosing microparticles. Here, we have addressed the hypothesis that microparticles trigger this process via lipid pathways. In these experiments, microparticles induced apoptosis in primary macrophage cells or cell lines (RAW 264.7 or U937) with up to a 5-fold increase. Preincubation of macrophages with phosphatidylinositol-3,5-bisphosphate (PtdIns(3,5)BP) reduced the microparticle-induced apoptosis in a dose-dependent manner. PtdIns(3,5)BP is a specific inhibitor of the acid sphingomyelinase and thus can block the generation of pro-apoptotic ceramides. Similarly, the preincubation of macrophages with $\operatorname{PtdIns}(3,5) \mathrm{BP}$ prevented microparticle-induced upregulation of caspase 8 , which is a major target molecule of ceramide action in the apoptosis pathway. PtdIns(3,5)BP, however, had no effect on the spontaneous rate of apoptosis. To evaluate further signaling pathways induced by microparticles, the extracellular signal regulated kinase (ERK-) 1 was investigated. This kinase
\end{abstract}

L. C. Huber $(\bowtie)$ A A Jüngel · J. H. W. Distler · F. Moritz ·

R. E. Gay · B. A. Michel - S. Gay · O. Distler

Center of Experimental Rheumatology, University Hospital

Zurich, Gloriastrasse 23, CH-8091, Zurich, Switzerland

and Zurich Center for Integrative Human Physiology (ZIHP)

e-mail: lars.huber@usz.ch

D. S. Pisetsky

Division of Rheumatology, Durham VA Hospital and Duke

University Medical Center,

Durham, NC, USA plays a role in activating phospholipases A2 which cleaves membrane phospholipids into arachidonic acid; microparticles have been suggested to be a preferred substrate for phospholipases A2. As shown in our experiments, microparticles strongly increased the amount of phosphorylated ERK1/2 in RAW 264.7 macrophages in a time-dependent manner, peaking 15 min after co-incubation. Addition of PD98059, a specific inhibitor of ERK1, prevented the increase in apoptosis of RAW 264.7 macrophages. Together, these data suggest that microparticles perturb lipid homeostasis of macrophages and thereby induce apoptosis. These results emphasize the importance of biolipids in the cellular cross-talk of immune cells. Based on the fact that in clinical situations with excessive cell death such as malignancies, autoimmune diseases and following chemotherapies high levels of circulating microparticles might modulate phagocytosing cells, a suppression of the immune response might occur due to loss of macrophages.

Keywords Microparticles · Membrane lipids .

Arachidonic acid $\cdot$ Apoptosis

\section{Introduction}

Microparticles are subcellular vesicles that are released from cells by shedding of membraneous blebs. The release of these structures is strongly increased when cells are exposed to proinflammatory or pro-apoptotic stimuli [1-3]. Consistent with a role of microparticles in the pathogenesis of inflammatory diseases, levels of these structures are elevated in autoimmune diseases such as rheumatoid arthritis [4], vasculitis [5], lupus anticoagulant syndrome [6], and multiple sclerosis [7]. These findings implicate microparticles as novel 
mediators of inflammation and suggest that the release of microparticles and their biological activities can be targeted in new approaches to immune modulation [8].

As shown by in vitro studies, microparticles can function as subcellular vectors to deliver proteins and lipids into target cells. This transfer can alter the phenotypic properties of the target cell as well as induce a variety of functional changes [9]. Recently, we have shown that microparticles derived from human Jurkat $T$-lymphoma cells are rapidly engulfed by RAW 264.7 mouse macrophages, which, in turn, undergo apoptosis [10]. The death of the macrophages leads to further generation of microparticles, albeit with different surface markers. While suggesting a mechanism for loss and functional disturbance of macrophages during inflammation, the mechanisms of apoptosis induced by the microparticles were not defined by these experiments.

In this current study, we have therefore explored this death pathway, focusing on the role of membrane phospholipids in the effects of microparticles on macrophage viability. In the induction of apoptosis, lipid metabolism has been implicated as a central event, with the pathway involving arachidonic acid and sphingomyelin receiving the most attention [11-15]. Arachidonic acid, which belongs to the group of essential twenty-carbon fatty acids (eicosanoids), is the most abundant polyunsaturated fatty acid among the phospholipid components of cell membranes. Arachidonic acid undergoes cleavage from membrane phospholipids by isoforms of phospholipase A2, which cleave the sn-2-acyl position of 1-ether-linked membrane glycerophospholipids to liberate fatty acids and lysophospholipids [16, 17].

The activity of phospholipase A2 requires a rearrangement of membrane lipids to expose phosphatidylserine on the external layer [18].

Shed from stimulated or dying cells, microparticles lose membrane asymmetry with subsequent surface exposure of aminophospholipids [19, 20]. These findings suggest that these structures could provide a substrate for phospholipases that is preferentially cleaved [21]. In this scenario, the accumulation of free arachidonic acid could enhance the activity of acid sphingomyelinase [22-25] to catalyze the conversion of sphingomyelin to cellular (C2/C16)-ceramides. With an increased concentration of ceramide, apoptosis would then be induced by the activation of caspase 8 and the release of downstream pro-apoptotic factors [26-28]. Of note, other phospholipases can degrade phospholipids more efficiently when sphingomyelinase is present and active [29-31].

Since microparticles consist of a packed phospholipid bilayer with altered membrane asymmetry, we have hypothesized that the transfer of a pool of 1-ether-linked phospholipids into target cells occurring during the phagocytosis of microparticles could perturb cellular arachidonic acid levels. With activated phospholipase A2, the changes in fatty acid metabolism could promote apoptosis in cells phagocytosing microparticles. We have therefore investigated the role of lipid mediators in the induction of macrophage apoptosis following interaction with microparticles and explored the effects of a specific inhibitor of syphingomyelinase. Results presented herein indicate the importance of this enzyme in the induction of apoptosis and the role of lipid mediators in cellular cross-talk stimulated by interaction of macrophages with microparticles.

\section{Materials and methods}

\section{Reagents}

Arachidonic acid as well as the pan-caspase-inhibitor $Z$-VAD-FMK were from Sigma (Sigma Chemical Co., St. Louis, MO), D-myo-phosphatidylinositol-3,5bisphosphate $[\operatorname{PtdIns}(3,5) \mathrm{P}]$ was purchased from Echelon Biosciences Incorporated (Salt Lake City, UT, USA). PD098059 (2'-amino-3'-methoxyflavone) was from Promega (Madison, WI, USA). Phycoerythrin (PE)labelled Annexin was from BD Biosciences Pharmingen (San Diego, CA, USA). JC-1 (5,5',6,6'-tetrachloro$1,1^{\prime}, 3,3^{\prime}$-tetraethylbenzimidazolylcarbocyanine Iodide) was purchased from Calbiochem (San Diego, CA, USA). Cell Signaling Cytobeads for ERK1/2 and JNK1/2 were from BD Biosciences Pharmingen.

\section{Cells/cell cultures}

Mouse macrophage cell line RAW 264.7 (adherent), human U937 (non-adherent), and Jurkat T lymphoma cells were purchased from ATCC (Manassas, VA) and cultured in Dulbecco's modified Eagle's medium (DMEM) (Gibco) containing $2 \mathrm{mmol} / \mathrm{l} L$-glutamine. DMEM was supplemented with $10 \%$ heat-inactivated fetal calf serum (FCS), $15 \mathrm{mmol} / \mathrm{l}$ HEPES, $50 \mu \mathrm{g} / \mathrm{ml}$ streptomycin, $100 \mathrm{U}$ of penicillin, and $2 \mu \mathrm{l} / \mathrm{ml}$ amphotericine B (all from Gibco).

For activation, Jurkat $T$ cells were stimulated with interleukin (IL-)2 (10 ng/ml) and U937 cells were stimulated with concanavalin $(1 \mu \mathrm{g} / \mathrm{ml}$; both from Sigma Chemical Co., St Louis, MO) in DMEM with 10\% FCS for $12 \mathrm{~h}$. After stimulation, the supernatants were collected and microparticles were isolated immediately as described previously [32]. For coculture experiments, $10^{5}$ confluent RAW 264.7 macrophages or $3 \times 10^{5}$ U937 cells were incubated for 6 and $36 \mathrm{~h}$ with freshly isolated microparticles from activated Jurkat $T$-cells.

The number of cells was determined by the CASY-1 cell counter (Schärfe System, Reutlingen, Germany).

Phagocytosis of microparticles by RAW macrophages was prevented by using anopore membrane systems (pore 
size $0.2 \mu \mathrm{m}$ ) as cell culture inserts (Nunc $\mathrm{GmbH}$, Wiesbaden, Germany), on which microparticles resuspended in PBS were added.

\section{Isolation of primary macrophages}

For the isolation of primary macrophages, buffy coats from healthy donors were obtained from the local blood bank. Peripheral blood mononuclear cells (PBMCs) were isolated by Ficoll/Hypaque (Pharmacia Biotech) density gradient centrifugation. After incubation with anti-human CD14microbeads (Miltenyi Biotec, Bergisch Gladbach, Germany), primary macrophages were isolated by positive selection magnetic cell sorting (MACS) according to the manufacture's instructions. After adding $50 \mathrm{ng} / \mathrm{ml} \mathrm{GM-CSF}$ at the initial day of culture, freshly isolated macrophages were cultured in modified Roswell Park Memorial Institute medium (RPMI 1640, with supplements as described above) for 7 days prior to incubation experiments.

\section{Isolation of Jurkat-derived microparticles}

Microparticles were prepared as described previously [32]. Briefly, cell culture supernatants obtained from Jurkat $T$-cells stimulated for $12 \mathrm{~h}$ were centrifuged at $1500 \mathrm{~g}$ for $10 \mathrm{~min}$ to remove suspended cells. Supernatants were then centrifuged twice at $100,000 \mathrm{~g}$ for $20 \mathrm{~min}$ at $14^{\circ} \mathrm{C}$ by using a Centrikon $T-1065$ centrifuge with a TST28.38 head (Kontron Instruments, Munich, Germany). Pelleted microparticles were resuspended in phosphate-buffered saline (PBS, pH 7.5). The microparticles were then either quantified and characterized by flow cytometry analysis or used for coculture experiments with RAW 264.7 or U937 cells. The number of microparticles as counted by FACS was adjusted to the number of RAW 264.7 or U937 cells, respectively.

\section{FACS}

Flow cytometry was performed using a FACScan flow cytometer (Becton Dickinson). A minimum of 10,000 cells per sample were acquired in list mode and analyzed using Cell quest software (Becton Dickinson Immunocytometry System, San Jose, CA).

\section{Detection of apoptosis}

Apoptosis was assessed by analyzing Annexin $V$-binding properties of membranes, measurement of the mitochondrial potential, and caspase 8 activity. Briefly, a total of $1 \times 10^{5}$ RAW macrophages and $3 \times 10^{5}$ human U937 cells were stimulated with $3 \times 10^{6}$ Jurkat-derived microparticles as described above. Inhibitor of acid sphingomyelinase was added in concentrations of 5 and $10 \mu \mathrm{M}$. Inhibition of general caspase activity was achieved by using $50 \mu \mathrm{M}$ of the pancaspase-inhibitor Z-VAD-FMK (Sigma). RAW macrophages were pre-incubated with Z-VAD-FMK for $1 \mathrm{~h}$ before adding microparticles. After 6 and $36 \mathrm{~h}$, the cells were then examined for apoptosis. In all apoptosis assays, negative controls consisted of untreated macrophages.

\section{Annexin staining}

$1 \times 10^{5}$ cells were examined for apoptosis using Annexin $V$ $\mathrm{PE}$ according to the manufacturer's protocol. Briefly, adherent cells were trypsinized, centrifuged at $500 \mathrm{~g}$ for $10 \mathrm{~min}$, and resuspended in $100 \mu \mathrm{l}$ of FACS binding buffer (BD Pharmingen, San Diego, CA, USA). Annexin $V$ - PE was added directly to the cells. After incubation for $15 \mathrm{~min}$ at room temperature, the cells were analyzed by FACS (FACScalibur, Becton Dickinson Mansfield, MA, USA).

Determination of mitochondrial membrane potential $(\Delta \varphi)$

$\Delta \varphi$ was analyzed by a previously described method which allows the measurement of $\Delta \varphi$ in intact cells [33, 34]. Briefly, JC-1 is a lipophilic cation that can selectively enter into mitochondria [35]. JC-1 exists in a monomeric form emitting at $527 \mathrm{~nm}$ after excitation at $490 \mathrm{~nm}$; however, depending on $\Delta \varphi, \mathrm{JC}-1$ is able to form $J$-aggregates that are associated with a large shift in emission $(590 \mathrm{~nm})$ [36, 37]. Thus, the color of the dye changes reversibly from green to greenish orange as the mitochondrial membrane becomes more polarized as a marker of late apoptosis [35]. Both colors can be detected using filters commonly mounted in flow cytometers, so that the green emission can be analysed in fluorescence channel 1 (FL1) and the greenish orange emission in channel 2 (FL2). Cell suspensions were incubated in complete medium with $\mathrm{JC}-1(5 \mu \mathrm{g} / \mathrm{ml})$ for $10 \mathrm{~min}$ at room temperature in the dark. At the end of the incubation period, the cells were washed once in cold phosphate-buffered saline (PBS), resuspended in a total volume of $400 \mu 1$ and analysed by FACS.

\section{Caspase 8 activity}

Cells were examined for apoptosis using a fluorimetric caspase 8 activity detection assay (Biovision, Mountain view, CA) according the manufacture's instructions. Briefly, cells were washed with PBS and resuspended in $50 \mu \mathrm{l}$ of Cell Lysis Buffer. After 10 min incubation period on ice, $50 \mu \mathrm{l}$ reaction buffer was added to the permeabilized cells. The cells were then stained with anti-active caspase 8 substrate (IETD-AFC), incubated for $2 \mathrm{~h}$ at $37^{\circ} \mathrm{C}$, and measured using a microtiter fluorescence reader plate (Witec AG, Biotec, Littau, Switzerland). Data were analyzed by using KC4 software (Bio-Tek Instruments, Vermont, USA). 
Inhibition of acid sphingomyelinase

Acid sphingomyelinase (aSMase) catalyzes the lysosomal degradation of sphingomyelin to phosphorylcholine and ceramide. Inhibition of aSMase was performed using $L$-alpha-phosphatidyl- $D$-myo-inositol-3,5-bisphosphate (PtdIns3, 5BP), a naturally occurring substance detected in mammalian, plant and yeast cells, which has been recently characterized [25]. Ptins3,5BP was used in concentrations of $2-10 \mu \mathrm{M}$, which is within the range of the ID50 of PTins3,5BP as described previously [25]. The effect on cell viability was determined by trypan blue exclusion. Briefly, respective concentrations of PtdIns3,5BP were added to U937 cells. After $6 \mathrm{~h}$ and $36 \mathrm{~h}, 50 \mu \mathrm{l}$ of cell suspensions were mixed with $50 \mu \mathrm{l}$ trypan blue and analysed using the Neubauer counting chamber. Compared to untreated U937 cells, there was no increase in the number of blue colored cells.

\section{Analysis of MAP-Kinases activation}

Phosphorylation of MAP-K (ERK1/2 and JNK 1/2) was determined by FACS analysis using Cell Signaling Flex Set assay procedure (BD Biosciences Pharmingen, San Diego, CA, USA) according the instructions of the manufacturer.

\section{Inhibition of MAP-Kinases (MEK1/ERK1)}

Inhibition of MEK1/ERK1 was performed using PD098059, a selective blocking agent of the activation of MEK1. PD098059 was used in concentrations of $10 \mu \mathrm{M}$, which is within the range of the ID50 of PD098059 as shown previously [38]. Trypan blue analysis of cytotoxicity after $6 \mathrm{~h}$ showed no effect on cell viability determined by cell counting.

\section{Statistical analysis}

All data are expressed as mean \pm standard deviation (SD). Statistical analysis was performed using the GraphPad Prism Software, 4.03 (Graph Pad Software Inc., San Diego, CA). For analysis between different groups the Mann-Whitney test was used, and a value of $p<0.05$ was considered statistical significant.

\section{Results}

Induction of apoptosis in macrophage cells by microparticles

Previous studies have shown that microparticles released from Jurkat $T$-cells are cleared by RAW 264.7 macrophages and, subsequently, induce apoptosis in these cells in a dosedependent manner [10]. Consistent with these findings, microparticles derived from Jurkat $T$-cells stimulated with IL-2 induced apoptosis in RAW 264.7 macrophages as determined by Annexin $V$ staining and FACS analysis $36 \mathrm{~h}$ after incubation. The frequency of spontaneous apoptotic cells in cultures of RAW 264.7 macrophages was $5.3 \pm 1.0 \%$, but this number was significantly increased to $40.2 . \pm 7.7 \%$ upon incubation with $3 \times 10^{6}$ Jurkat-derived microparticles. In these experiments, the number of microparticles used was within the physiological range of levels of circulating microparticles reported previously $[4,39]$.

To evaluate whether human macrophages also undergo apoptosis after incubation with microparticles, microparticles from Jurkat cells were added to $3 \times 10^{5}$ human U937 cells as well as $3 \times 10^{5}$ primary macrophages from healthy donors. In both conditions, apoptosis was strongly induced in macrophages $36 \mathrm{~h}$ after incubation with microparticles. In human U937 cells, the induction of apoptosis was less prominent (by about 3 fold from $12.0 \pm 3.6 \%$ to $35.3 \pm 6.0 \%$ ); this difference may relate to the lower phagocytotic capacity of these myeloid precursor cells (Fig. 1). In contrast, cells without phagocytosing capacity such as dermal and synovial fibroblasts derived from different diseases did not show an increased rate of apoptosis upon treatment with microparticles (data not shown). In a next step, we used cell culture inserts as microfilters to prevent direct contact and phagocytosis of microparticles by RAW macrophages. Anopore filters of $0.2 \mu \mathrm{m}$ strongly reduced the microparticle-induced apoptosis by $53.3 \pm 4.3 \%$ after $36 \mathrm{~h}$ as measured by FACS analysis using Annexin $V$-PE. These data suggest that either direct cell contact and most likely, phagocytosis, is required to induce apoptosis in RAW 264.7 macrophages by microparticles. These results were further confirmed by incubating macrophages with microparticle-free supernatant that did not change the rate of apoptosis of target cells.

To explore further mechanisms of macrophage apoptosis in this system, we first analyzed whether microparticles from various origins can induce apoptosis in target cells to an equivalent extent. We therefore stimulated both $\mathrm{Ju}$ rkat $T$-cells and human U937 cells to induce the production of microparticles. These particles were isolated after $12 \mathrm{~h}$ and then added at a concentration of $3 \times 10^{6}$ to $10^{5}$ RAW 264.7 macrophages. Six hours after incubation, apoptosis was measured by FACS analysis using JC-1. With microparticles from both sources, a strong shift of the fluorescent signal from greenish-orange (fluorescence (FL-) 1 and FL-2 double-positive) to green (FL1 single-positive) could be observed. This shift was due to depolarising changes of the mitochondrial membrane potential, indicating apoptotic events in microparticle-stimulated cells compared to untreated control macrophages. Microparticles from $3 \times 10^{6}$ Jurkat T-cells 


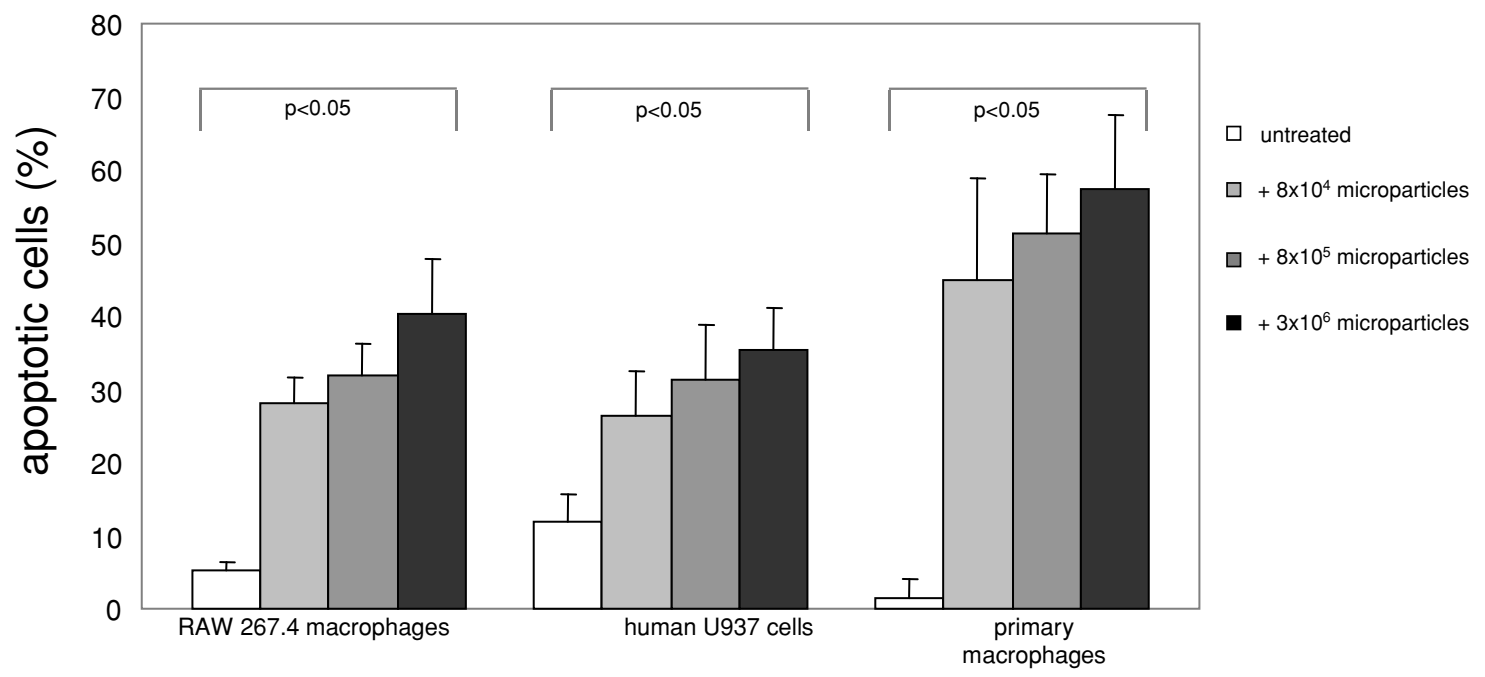

Fig. 1 Induction of apoptosis in RAW 264.7 macrophages upon stimulation with Jurkat-derived microparticles. In untreated RAW 264.7 cells (white bar), a spontaneous apoptosis rate of $5.3 \pm 1.0 \%$ was measured. When incubated with $3 \times 10^{6}$ Jurkat-derived microparticles,
RAW 264.7 macrophages showed an increased apoptosis rate of $40.2 \pm 7.7 \%$ (black bar). Similar results were observed in human 937 cells and in primary macrophages. The apoptosis rate was measured by FACS analysis using Annexin V-PE increased the apoptotic RAW 264.7 cell population from 5\% to $14.5 \pm 4.4 \%$ (Fig. 2a), while microparticles from U937 cells increased apoptosis from $5 \%$ to $22.0 \pm 1.8 \%$ (Fig. 2b).

The effect of inhibiting acid sphingomyelinase on microparticle-induced apoptosis

Following cell stimulation, isoforms of phospholipase A2 can cleave membrane phospholipids to liberate arachidonic acid, which, in turn, activates the enzyme acid sphingomyelinase (aSMase) to metabolize sphingomyelin into ceramides. To investigate whether microparticles activate the apoptotic program in phagocytes through the arachidonic acid pathway, RAW 264.7 macrophages were incubated with different concentrations of arachidonic acid for $36 \mathrm{~h}$ before analysis of apoptosis. As shown in Fig. 3, arachidonic acid dose-dependently induced apoptosis in macrophages, with maximum effects observed at $100 \mu \mathrm{M}$.

To assess the down-stream effects of archidonic acid, RAW 264.7 macrophages were next pre-incubated for $2 \mathrm{~h}$ with varying concentrations of the specific aSMase-inihibitor phosphatidylinositol-3,5-biphosphate [Ptins(3,5)BP] [25] before adding microparticles. Apoptosis was then tested. Figure 4 shows the effects of Ptins(3,5)BP on macrophages that were incubated for 6 and $36 \mathrm{~h}$ with microparticles. In these experiments, Ptins(3,5)BP decreased the apoptosisinducing effect of microparticles on macrophages in a dosedependent manner. Six hours after incubation, Ptins(3,5)BP reduced the fraction of cells with a depolarised mitochondrial potential by $36.5 \pm 7.6 \%(5 \mu \mathrm{M})$ and by $66 \pm 17.8 \%(10 \mu \mathrm{M}$; $p<0.05)$ respectively. Similarly, FACS analysis performed after $36 \mathrm{~h}$ showed that the depolarisation of the mitochondrial potential as seen in early apoptosis could be prevented by adding Ptins(3,5)BP to RAW 264.7 macrophages that were incubated with Jurkat — and U937-derived microparticles. The higher dose of this inhibition produced statistically significant inhibition in both experimental conditions. In addition, RAW macrophages were preincubated with the general caspase inhibitor Z-VAD-FMK before microparticles were added. Rates of apoptosis were reduced, in particular when analysed after $6 \mathrm{~h}$ (Fig. 4). These data indicate that the activation of the phospholipide-ceramide pathway by microparticles results in both caspase-dependent and-independent cell death with caspases mainly involved during the early stages of apoptosis.

The effect of Ptins(3,5)BP on the basal rate of apoptosis

Untreated U937 cells showed a frequency of Annexin Vpositive cells of $12.0 \pm 3.6 \%$ after $36 \mathrm{~h}$ of culture. To exclude the possibility that the observed reduction in the Annexin Vpositive population after co-incubation with microparticles and Ptins(3,5)BP resulted from a reduction of the spontaneous rate of apoptosis, U937 cells were incubated with medium alone or in combination with different concentrations of Ptins(3,5)BP. Staining with Annexin V and FACS analysis after $36 \mathrm{~h}$ showed no differences in the intensity of the fluorescent signals between untreated cells and aSMaseinhibited cells at any concentration. In addition, the treatment with Ptins(3,5)BP had no toxic effects on cells at the concentrations used in the study, as measured by trypan blue exclusion (see experimental procedures). 

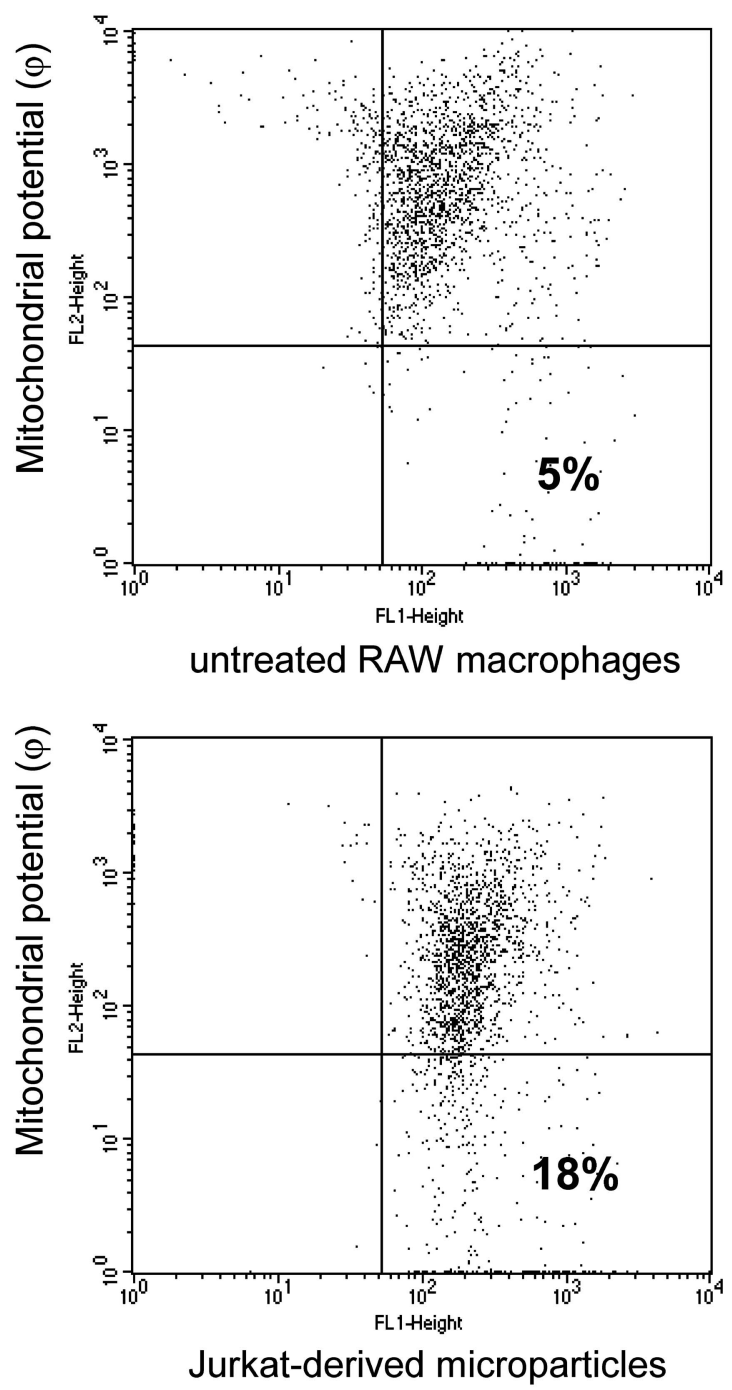

(a)

Fig. 2 Induction of apoptosis in RAW 264.7 macrophages upon stimulation with microparticles. RAW macrophages were incubated with microparticles from Jurkat T-cells and human U937 cells. Apoptosis rate was measured by FACS analysis after $6 \mathrm{~h}$ using JC- 1 . The upper panels show the mitochondrial membrane potential of untreated control cells. The lower panels are as follows: (a) changes in the mito-

The effects of microparticles on caspase 8 activity in macrophage target cells

Arachidonic acid activates the enzyme acid sphingomyelinase (aSMase) leading to the generation of ceramides. A major target molecule of ceramide action is caspase 8 , which, in turn, cleaves the pro-apoptotic factor bid into its active conformation. The next series of experiments investigated the role of microparticles in downstream activation of caspase 8 . Stimulation of RAW 264.7 macrophages with Jurkat-derived microparticles showed a strong activation of caspase 8 as determined by fluorimetric analysis after $36 \mathrm{~h}$ (Fig. 5). Basal
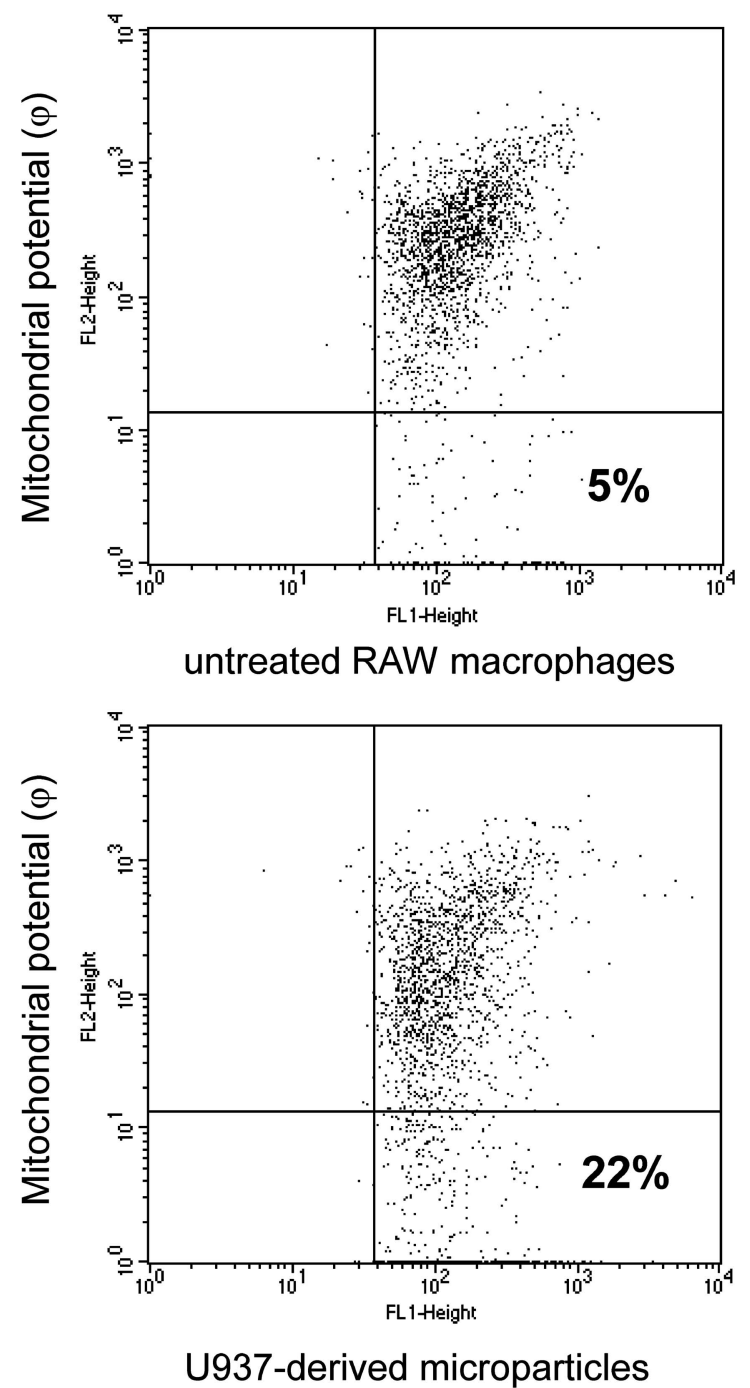

(b)

chondrial potential $(\Delta \varphi)$ indicating an increase of apoptosis upon incubation with Jurkat-derived microparticles from $5 \%$ to $14.5 \pm 4.4 \%$; (b) changes in the mitochondrial potential $(\Delta \varphi)$ indicating an increase of apoptosis upon incubation with U937-derived microparticles from 5\% to $22.0 \pm 1.8 \%$

rate was determined by measuring the caspase 8 activity of unstimulated cells.

To explore these mechanisms further, we investigated the effect of Ptins $(3,5) \mathrm{BP}$ on $10^{5}$ RAW 264.7 macrophages when co-incubated with $3 \times 10^{6}$ Jurkat-derived microparticles. The addition of microparticles increased the basal enzymatic activity of caspase 8 by $33 \pm 6 \%$ from $3568 \pm 5$ relative fluorescence units (RFU) to $4517 \pm 621$. Similar to the results of our previous experiments, Ptins $(3,5) \mathrm{BP}$ prevented the activation of caspase 8 in a dose-dependent manner. Co-incubation of macrophages with microparticles and PtdIns(3,5)BP showed a caspase 8 activity of $4060 \pm 368$ 
Fig. 3 Effects of arachidonic acid on apoptosis of macrophages. Arachidonic acid dose-dependently induced apoptosis in RAW macrophages. Macrophages were incubated with medium alone (white bar), or incubated with different concentrations of arachidonic acid (1-100 $\mu \mathrm{M})$. Changes of the mitochondrial potential were measured by FACS using JC-1 after $36 \mathrm{~h}$

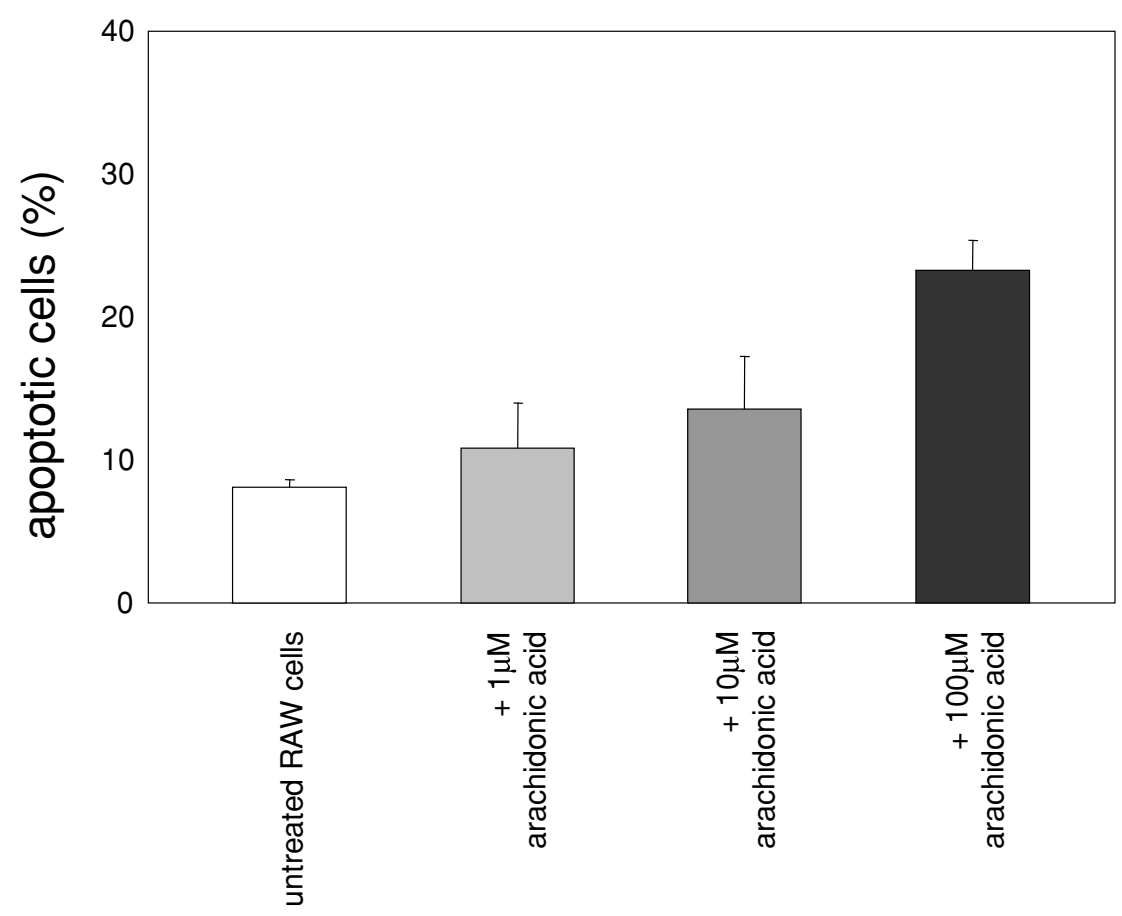

Fig. 4 Inhibitory effects of Ptins $(3,5) \mathrm{BP}$ on microparticle-induced apoptosis. Ptins $(3,5) \mathrm{BP}$ prevented the microparticle-induced depolarisation of the mitochondrial membrane in a dose dependent manner. Phagocytes were incubated with medium alone (white bars), or incubated with $3 \times 10^{6}$ microparticles (black bars) and treated with different concentrations of Ptins $(3,5) \mathrm{BP}$. Preincubation with the pan-caspase inhibitor Z-VAD-FMK $(50 \mu \mathrm{M})$ before adding microparticles (dashed bars). Changes of the mitochondrial potential were measured by FACS using JC-1 after 6 (left side) and $36 \mathrm{~h}$ (right side) 


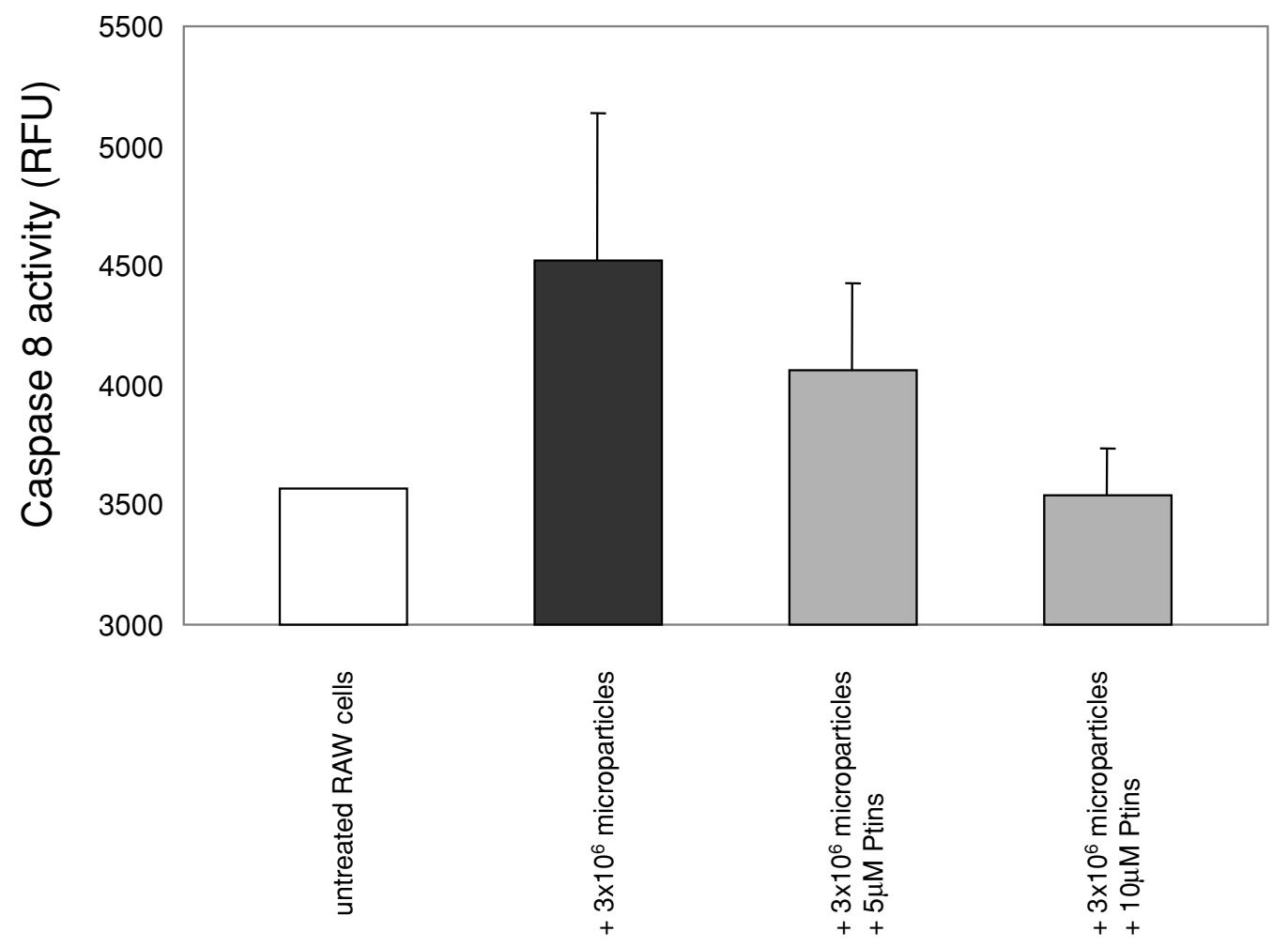

Fig. 5 Ptins (3, 5)BP prevents microparticle-induced up-regulation of caspase 8 . The basal activity of caspase 8 was determined in untreated macrophages and reached a level of $3500 \pm 5$ relative fluorescence units (RFU). Addition of $3 \times 10^{6}$ microparticles increased caspase 8

RFU by using $5 \mu \mathrm{M}$ and $3539 \pm 196 \mathrm{RFU}$ by using $10 \mu \mathrm{M}$ PtdIns $(3,5) \mathrm{BP}(p<0.05)$, respectively.

The effects of microparticles on MAP-Kinases

Microparticles have been suggested to be a preferred substrate for phospholipases A2, which cleave membrane phospholipids into arachidonic acid [14]. Several members of the MAP-Kinase pathway, in particular the extracellular signal regulated kinase (ERK-)1, play an important role in activating phospholipases A2. Thus, we tested the effects of microparticles on these downstream signalling events. When analysed by FACS using Cytobeads, $3 \times 10^{6}$ Jurkatderived microparticles strongly increased the amount of phosphorylated ERK1/2 in RAW 264.7 macrophages from $63.2 \pm 30.4 \%$ to $286.6 \pm 51.7 \%$ in a time-dependent manner, peaking 15 minutes after co-incubation (Fig. 6). Similar results were observed for other members of the MAPKinase pathway (i.e. JNK1/2), although to a lesser extent. Subsequently, we added PD98059, a specific inhibitor of ERK1, to RAW macrophages exposed to microparticles. PD98059 resulted in an abrogation $(27.7 \pm 4.2 \%)$ of the apoptosis-promoting effects of Jurkat-derived microparticles in RAW 264.7 cells as assessed $6 \mathrm{~h}$ after incubation by FACS analysis.

activity by $33 \pm 6 \%$ to $4517 \pm 621 \mathrm{RFU}$, whereas pre-incubation of macrophages with PtdIns $(3,5) \mathrm{BP}$ prevented the microparticle-induced up regulation of caspase 8 after $36 \mathrm{~h}$ in a dose-dependent manner (4060 \pm 368 by using $5 \mu \mathrm{M}$, and $3539 \pm 196 \mathrm{RFU}$ by using $10 \mu \mathrm{M}$ )

To exclude the possibility that the observed reduction in the Annexin V-positive population after co-incubation with microparticles and PD098059 resulted from a reduction of the spontaneous rate of apoptosis, U937 cells were incubated with various concentrations of PD098059 alone. Analysis of Annexin $\mathrm{V}$ positive cells after $6 \mathrm{~h}$ showed no differences between untreated cells and MEK1/ERK1-inhibited cells. Furthermore, the treatment with Ptins(3,5)BP had no effects on cell viability at the concentrations used in the study, as measured by trypan blue exclusion (see experimental procedures). Together, these findings indicate a role of ceramide acting via MAP-Kinases in the induction of apoptosis by microparticles.

\section{Discussion}

Results of these studies provide new insight into the biological activities of microparticles and demonstrate a role of lipids in the apoptosis-inducing effects of these structures. Thus, we have shown that microparticles preparations derived from activated cell lines induce the apoptosis program in RAW 264.7 macrophages [10]. This induction occurred with microparticles derived both Jurkat and U937 cells, suggesting a common mechanism for the induction of apoptosis. 
Fig. 6 Activation of MAP-Kinases by microparticles. Basal levels of phosphorylated ERK1/2 and JNK $1 / 2$ were measured in untreated RAW macrophages. Addition of $3 \times 10^{6}$ Jurkat-derived microparticles increased levels of phosphorylated ERK1/2 from $63.2 \pm 30.4 \%$ to $286.6 \pm 51.7 \%$ (mean fluorescence intensity) in a time-dependent manner. Similar effects were observed for JNK $1 / 2$

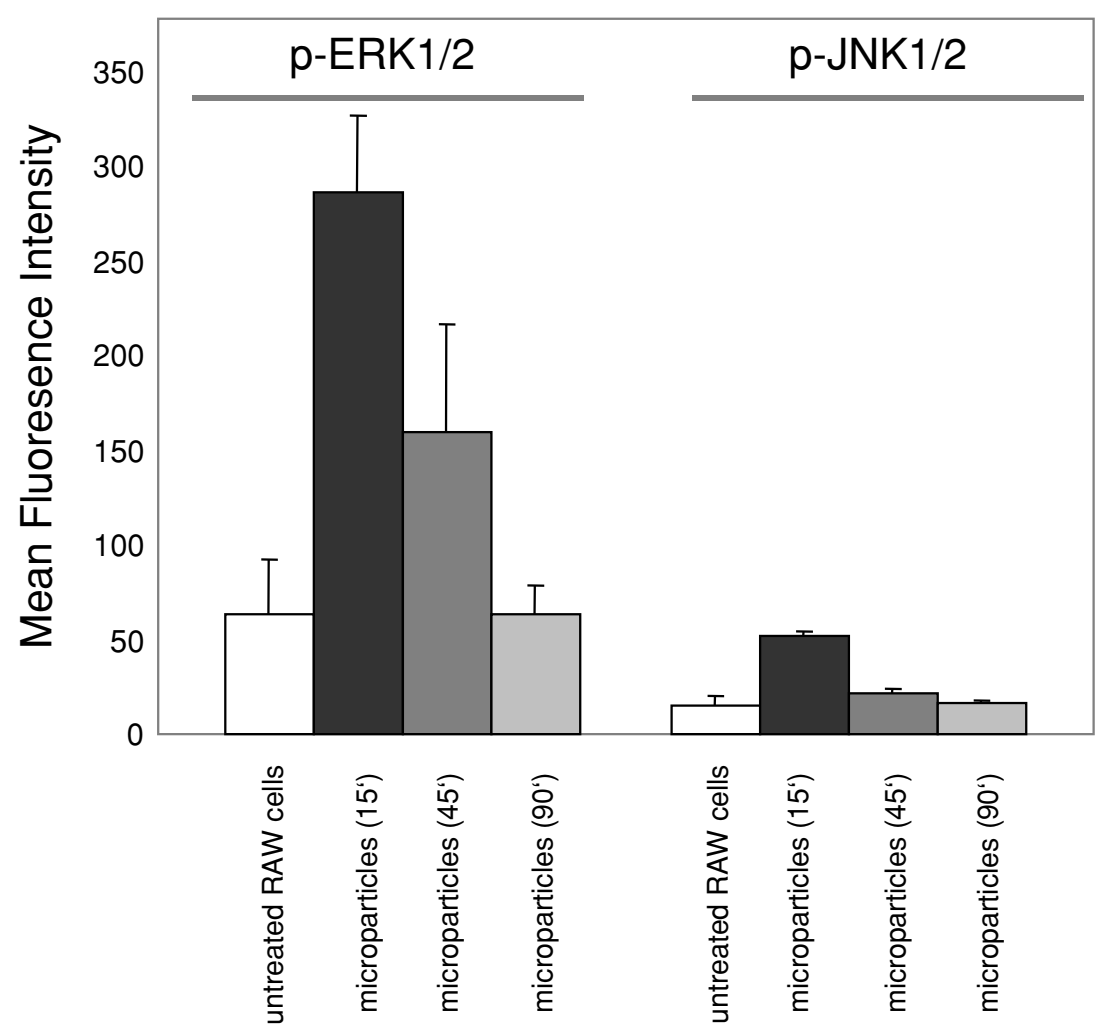

Since microfilters strongly reduced the microparticleinduced apoptosis, cell-cell contact, most likely involving phagocytosis, may play an important role for the apoptosis response. This possibility is further supported by the observation that cells with a low phagocytic capacity did not undergo apoptosis under conditions in which macrophage cell lines demonstrated this response. Since cell death was inhibited completely by the addition of microparticle-free supernatant but not by using microfilters, however, it is possible that, during culture, microparticles release fragments with membraneous phospholipids which may pass the filter to interact with macrophages due to their smaller size.

Together with previous observations on the activity of microparticles, these findings suggest that microparticles induce apoptosis by a dysregulation of phospholipidarachidonate pathway rather than interaction with a specific surface receptor. Microparticles represent a population of vesicles of 200-700 nm size surrounded by a packed phospholipid bilayer originating from the cell membranes. These particles can interact with, and enter target, cells during phagocytosis. In this process, a rapid redistribution of arachidonate from releasable pools by phospholipases A2 may increase the levels of free unesterified arachidonic acid. This lipid mediator appears to be an important signal both for inhibiting cell proliferation and inducing apoptosis by regulating gene expression, controlling transcription factors, and generation of inflammatory mediators [40-42].
Among its actions, arachidonic acid can activate acid sphingomyelinase to metabolize sphingomyelin into $\mathrm{C} 2 / \mathrm{C} 16$ ceramides and, thereby, initiate the apoptotic cascade. Studies on a variety of systems indicate that maintenance of arachidonate levels is important for homeostasis and a necessary condition for cell growth and survival. It is of interest, in this regard, that cancer cells depleted of arachidonate display an increased resistance to apoptosis. Furthermore, the intracellular accumulation of arachidonic acid following treatment with nonsteroidal anti-inflammatory drugs may be beneficial in treatment of colorectal cancer by leading to the generation of ceramides and, ultimately, the induction of apoptosis [11, 14].

Using phosphatidylinositol-3,5-biphosphate (Ptins(3, 5)BP), a specific inhibitor of the arachidonate-dependent enzyme acid sphingomyelinase, we identified sphingomyelin as a key mediator in microparticle-induced apoptosis. Thus, in our experiments, Ptins(3,5)BP reduced the occurrence of apoptosis in microparticle-treated macrophage cells in a dose-dependent manner without affecting the spontaneous rate of apoptosis in these cells. To clarify further the mechanisms of this response, we investigated the role of MAPKinases. These kinases could play a role in apoptosis if, during particle interaction with the target cell, membrane phospholipids from microparticles are cleaved by the enzyme phospholipase A2. In this context, the cytosolic isoform of phospholipase $A_{2}$ shows a strong preference for sn2 bond arachidonic acid over other fatty acids and appears 


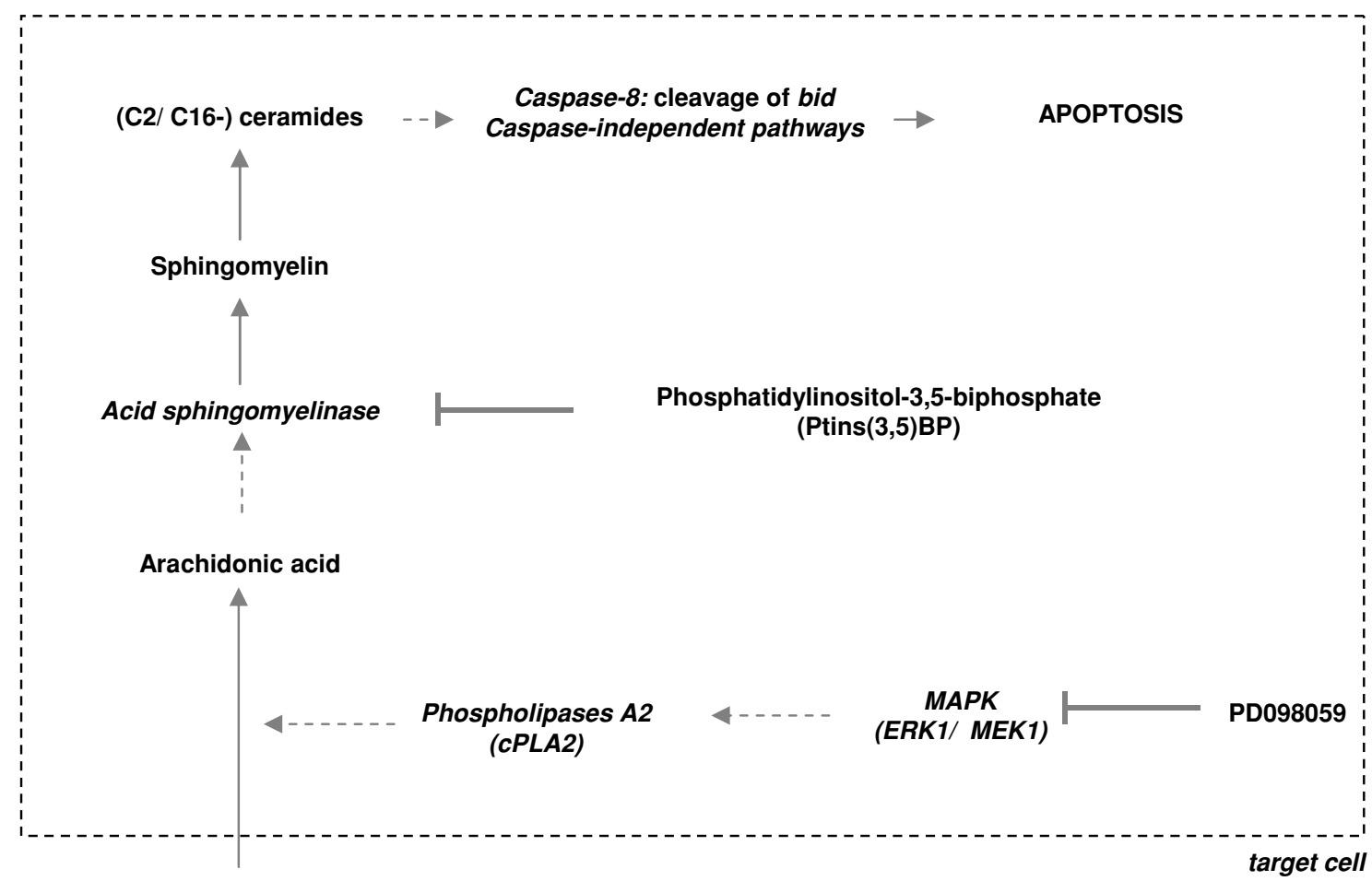

s2n-acyl ester bonds in phospholipids

- microparticles

- membrane fragments

Fig. 7 Proposed mechanism of apoptosis-induction of microparticles in phagocytosing cells by phospholipid-arachidonate-ceramide remodeling. The phagocytosis of microparticles leads to an increased cellular content of 1-ether-linked membrane phospholipids, which are cleaved by phospholipases A2 into arachidonic acid. Arachidonic acid

to mediate stimulus-coupled release of arachidonate during inflammatory processes [43]. Furthermore, serine phosphoacceptor sites, which are phosphorylated by MAP-Kinases, are essential for the stimulus-coupled activation of cytosolic phospholipase $\mathrm{A}_{2}[44,45]$.

As shown in these experiments, microparticles strongly induced the phosphorylation of ERK1/2, a tyrosine kinase central to the operation of the MAP-Kinase pathway, in a time-dependent manner. In addition, the specific MAPKinase inhibitor PD098059 caused a marked reduction in the population of early apoptotic cells induced by treatment of macrophages with microparticles, further supporting the role of this pathway. Based on these results, we propose that microparticles induce apoptosis in phagocytes by disturbing the lipid content of cells, in particular, by increasing the concentration free arachidonic acid (Fig. 7). Arachidonic acid in turn can stimulate the activity of acid sphingomyelinase to generate ceramides that trigger apoptosis. Since mi-

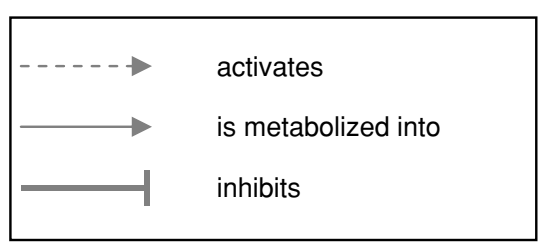

is a strong activator of acid sphingomyelinase that metabolizes sphingomyelin in pro-apoptotic ceramides. Blocking this cycle by inhibition of ERK1/MEK1 (PD98059) or by inhibition of the acid sphingomyelinase $[$ Ptins $(3,5) \mathrm{BP}]$ abrogates the microparticle-induced apoptosis response in target cells

croparticles can activate this response, it appears likely that these structures provide phospholipids substrates that are preferentially cleaved by phospholipases to provide a source of arachidonate. This preference may result from the subcellular location of the particles during phagocytosis or other cellular changes occurring during this process.

As reported previously [10], in clinical situations with high levels of circulating microparticles or excessive apoptosis such as malignancies, autoimmune syndromes and chemotherapy, microparticles might set off an amplification loop of apoptosis in phagocytosing cells thus suppressing the immune response by loss of macrophages. By describing an important intracellular pathway of this mechanism, our results might help to identify novel therapeutic strategies to overcome complications of these severe clinical entities.

In summary, these studies demonstrate that microparticles from immune cells can induce apoptosis in macrophage cell lines as well as primary macrophages. This process can be 
inhibited by interfering with the phospholipid-arachidonate remodelling, suggesting that the apoptotic cascade induced by interaction of microparticles with phagocytosing cells is mediated by the arachidonate-ceramide pathway. We have suggested previously that the ingestion of microparticles may represent a novel and specific mechanism for the induction of apoptosis in macrophages that could be operative in a number of clinical settings, including sepsis, systemic inflammation and malignancy. In these settings, the extent of microparticle release may increase, thereby causing subsequent immune system derangement [10]. Here, we propose a molecular mechanism underlying this phenomenon. Moreover, our results emphasize the importance of lipids in the cross-talk of immune cells that can operate with both intact cells as well as subcellular vesicles. Future studies will investigate the potency of these particles as a source of membrane lipids that can trigger cellular responses.

Acknowledgment This research was supported by the Swiss National Science Foundation (SNSF)-Grant 3200BO-103691 as well as by the European Community's FP6 funding. This publication reflects only the author's view. The European Community is not liable for any use that may be made of the information herein.

\section{References}

1. Freyssinet JM (2003) Cellular microparticles: what are they bad or good for? J Thromb Haemost 1:1655-1662

2. Aupeix K, Hugel B, Martin T, Bischoff P, Lill H, Pasquali JL, Freyssinet JM (1997) The significance of shed membrane particles during programmed cell death in vitro, and in vivo, in HIV-1 infection. J Clin Invest 99:1546-1554

3. Zhang J, Reedy MC, Hannun YA, Obeid LM (1999) Inhibition of caspases inhibits the release of apoptotic bodies: Bcl-2 inhibits the initiation of formation of apoptotic bodies in chemotherapeutic agent-induced apoptosis. J Cell Biol 145:99-108

4. Berckmans RJ, Nieuwland R, Tak PP, Boing AN, Romijn FP, Kraan MC, Breedveld FC, Hack CE, Sturk A (2002) Cell-derived microparticles in synovial fluid from inflamed arthritic joints support coagulation exclusively via a factor VII-dependent mechanism. Arthritis Rheum 46:2857-2866

5. Brogan PA, Shah V, Brachet C, Harnden A, Mant D, Klein N, Dillon MJ (2004) Endothelial and platelet microparticles in vasculitis of the young. Arthritis Rheum 50:927-936

6. Combes V, Simon AC, Grau GE, Arnoux D, Camoin L, Sabatier F, Mutin M, Sanmarco M, Sampol J, Dignat-George F (1999) In vitro generation of endothelial microparticles and possible prothrombotic activity in patients with lupus anticoagulant. J Clin Invest 104:93-102

7. Minagar A, Jy W, Jimenez JJ, Sheremata WA, Mauro LM, Mao WW, Horstman LL, Ahn YS (2001) Elevated plasma endothelial microparticles in multiple sclerosis. Neurology 56:1319-1324

8. Distler JH, Pisetsky DS, Huber LC, Kalden JR, Gay S, Distler O (2005) Microparticles as regulators of inflammation: novel players of cellular crosstalk in the rheumatic diseases. Arthritis Rheum 52:3337-3348

9. Barry OP, Pratico D, Savani RC, FitzGerald GA (1998) Modulation of monocyte-endothelial cell interactions by platelet microparticles. J Clin Invest 102:136-144

10. Distler JH, Huber LC, Hueber AJ, Reich CF, 3rd, Gay S, Distler O, Pisetsky DS (2005) The release of microparticles by apoptotic cells and their effects on macrophages. Apoptosis 10:731-741

11. Surette ME, Fonteh AN, Bernatchez C, Chilton FH (1999) Perturbations in the control of cellular arachidonic acid levels block cell growth and induce apoptosis in HL-60 cells. Carcinogenesis 20:757-763

12. Hollenbach PW, Zilli DL, Laster SM (1992) Inhibitors of transcription and translation act synergistically with tumor necrosis factor to cause the activation of phospholipase A2. J Biol Chem 267:39-42

13. Tang DG, Chen YQ, Honn KV (1996) Arachidonate lipoxygenases as essential regulators of cell survival and apoptosis. Proc Natl Acad Sci USA 93:5241-5246

14. Tsujii M, DuBois RN (1995) Alterations in cellular adhesion and apoptosis in epithelial cells overexpressing prostaglandin endoperoxide synthase 2. Cell 83:493-501

15. Chang J, Musser JH, McGregor H (1987) Phospholipase A2: function and pharmacological regulation. Biochem Pharmacol 36:2429-2436

16. Murakami M, Masuda S, Kudo I (2003) Arachidonate release and prostaglandin production by group IVC phospholipase A2 (cytosolic phospholipase A2gamma). Biochem J 372:695-702

17. Murakami M, Kudo I (2001) Diversity and regulatory functions of mammalian secretory phospholipase A2s. Adv Immunol 77:163194

18. Kudo I, Murakami M, Hara S, Inoue K (1993) Mammalian nonpancreatic phospholipases A2. Biochim Biophys Acta 1170:217231

19. Zwaal RF, Schroit AJ (1997) Pathophysiologic implications of membrane phospholipid asymmetry in blood cells. Blood 89:11211132

20. Sims PJ, Wiedmer T (2001) Unraveling the mysteries of phospholipid scrambling. Thromb Haemost 86:266-275

21. Fourcade O, Simon MF, Viode C, Rugani N, Leballe F, Ragab A, Fournie B, Sarda L, Chap H (1995) Secretory phospholipase A2 generates the novel lipid mediator lysophosphatidic acid in membrane microvesicles shed from activated cells. Cell 80:919_ 927

22. Obeid LM, Linardic CM, Karolak LA, Hannun YA (1993) Programmed cell death induced by ceramide. Science 259:1769-1771

23. Obeid LM, Hannun YA (1995) Ceramide: a stress signal and mediator of growth suppression and apoptosis. J Cell Biochem 58:191198

24. Jayadev S, Linardic CM, Hannun YA (1994) Identification of arachidonic acid as a mediator of sphingomyelin hydrolysis in response to tumor necrosis factor alpha. J Biol Chem 269:57575763

25. Kolzer M, Arenz C, Ferlinz K, Werth N, Schulze H, Klingenstein R, Sandhoff K (2003) Phosphatidylinositol-3,5-Bisphosphate is a potent and selective inhibitor of acid sphingomyelinase. Biol Chem 384:1293-1298

26. Di Paola M, Zaccagnino P, Montedoro G, Cocco T, Lorusso M (2004) Ceramide induces release of pro-apoptotic proteins from mitochondria by either a $\mathrm{Ca}^{2+}$-dependent or a $\mathrm{Ca}^{2+}$-independent mechanism. J Bioenerg Biomembr 36:165-170

27. Kannan R, Jin M, Gamulescu MA, Hinton DR (2004) Ceramideinduced apoptosis: role of catalase and hepatocyte growth factor. Free Radic Biol Med 37:166-175

28. Lin CF, Chen CL, Chang WT, Jan MS, Hsu LJ, Wu RH, Tang MJ, Chang WC, Lin YS (2004) Sequential caspase-2 and caspase-8 activation upstream of mitochondria during ceramideand etoposideinduced apoptosis. J Biol Chem 279:40755-40761

29. Verkleij AJ, Zwaal RF, Roelofsen B, Comfurius P, Kastelijn D, van Deenen LL (1973) The asymmetric distribution of phospholipids in the human red cell membrane. A combined study using phospholipases and freeze-etch electron microscopy. Biochim Biophys Acta 323:178-193 
30. Zwaal RF, Roelofsen B, Comfurius P, van Deenen LL (1975) Organization of phospholipids in human red cell membranes as detected by the action of various purified phospholipases. Biochim Biophys Acta 406:83-96

31. Chap HJ, Zwaal RF, van Deenen LL (1977) Action of highly purified phospholipases on blood platelets. Evidence for an asymmetric distribution of phospholipids in the surface membrane. Biochim Biophys Acta 467:146-164

32. Distler JH, Jungel A, Huber LC, Seemayer CA, Reich CF, 3rd, Gay RE, Michel BA, Fontana A, Gay S, Pisetsky DS, Distler O (2005) The induction of matrix metalloproteinase and cytokine expression in synovial fibroblasts stimulated with immune cell microparticles. Proc Natl Acad Sci USA 102:2892-2897

33. Polla BS, Kantengwa S, Francois D, Salvioli S, Franceschi C, Marsac C, Cossarizza A (1996) Mitochondria are selective targets for the protective effects of heat shock against oxidative injury. Proc Natl Acad Sci USA 93:6458-6463

34. Cossarizza A, Baccarani-Contri M, Kalashnikova G, Franceschi C (1993) A new method for the cytofluorimetric analysis of mitochondrial membrane potential using the Jaggregate forming lipophilic cation 5, , $5^{\prime}, 6,6^{\prime}$-tetrachloro-1, $1^{\prime}, 3,3^{\prime}$ tetraethylbenzimidazolcarbocyanine iodide (JC-1). Biochem Biophys Res Commun 197:40-45

35. Smiley ST, Rudensky AY, Glimcher LH, Grusby MJ (1996) Truncation of the class II beta-chain cytoplasmic domain influences the level of class II/invariant chain-derived peptide complexes. Proc Natl Acad Sci USA 93:241-244

36. Hada H, Honda C, Tanemura H (1977) Spectroscopic study on the J-aggregate of cyanine dyes. Photogr Sci Eng 21:83-91

37. Reers M, Smith TW, Chen LB (1991) J-aggregate formation of a carbocyanine as a quantitative fluorescent indicator of membrane potential. Biochemistry 30:4480-4486
38. Watts SW (1998) Activation of the mitogen-activated protein kinase pathway via the 5-HT2A receptor. Ann NY Acad Sci 861:162168

39. Guiducci S, Distler JH, Jungel A, Huber LC, Michel BA, Gay RE, Kalden J, Pisetsky DS, Matucci-Cerinic M, Gay S, Distler O (2005) Elevated numbers of microparticles in the blood of patients with systemic sclerosis. Arthritis Rheum 52: S461

40. Cowlen MS, Eling TE (1993) Effects of prostaglandins and hydroxyoctadecadienoic acid on epidermal growth factor-dependent DNA synthesis and c-myc proto-oncogene expression in Syrian hamster embryo cells. Biochim Biophys Acta 1174:234-240

41. Jurivich DA, Sistonen L, Sarge KD, Morimoto RI (1994) Arachidonate is a potent modulator of human heat shock gene transcription. Proc Natl Acad Sci USA 91:2280-2284

42. Rao GN, Baas AS, Glasgow WC, Eling TE, Runge MS, Alexander RW (1994) Activation of mitogen-activated protein kinases by arachidonic acid and its metabolites in vascular smooth muscle cells. J Biol Chem 269:32586-32591

43. Clark JD, Lin LL, Kriz RW, Ramesha CS, Sultzman LA, Lin AY, Milona N, Knopf JL (1991) A novel arachidonic acid-selective cytosolic PLA2 contains a $\mathrm{Ca}(2+)$-dependent translocation domain with homology to PKC and GAP. Cell 65:1043-1051

44. Lin LL, Wartmann M, Lin AY, Knopf JL, Seth A, Davis RJ (1993) cPLA2 is phosphorylated and activated by MAP kinase. Cell 72:269-278

45. Hefner Y, Borsch-Haubold AG, Murakami M, Wilde JI, Pasquet S, Schieltz D, Ghomashchi F, Yates JR, 3rd, Armstrong CG, Paterson A, Cohen P, Fukunaga R, Hunter T, Kudo I, Watson SP, Gelb MH (2000) Serine 727 phosphorylation and activation of cytosolic phospholipase A2 by MNK1-related protein kinases. J Biol Chem 275:37542-37551 